Wie sehr diese Behauptung den wirklichen in der Praxis bestehenden Verhältnissen zuwiderläuft, wird am einfachsten durch den Hinweis auf die Befunde an den Nudeln No. IV, V und VI bewiesen. Ich war in der Lage, über die Zeit der Herstellung der Muster einwandfreie Auskünfte zu erlangen. Die Nudeln No. V und VI - aus Kleinbetrieben stammend - waren bei der Einlieferung noch knetbar und biegsam und 2 bezw. 3 Tage alt, die Nudel No. IV war 14 Tage alt, als sie zur Untersuchung eingeliefert wurde. Alle drei sind demgemäß frische Waren. Ein Blick auf die während der $2 / 3^{-}$bis einjährigen Lagerung eingetretenen Veränderungen der Lecithinphosphorsäure lehrt, daß der Rückgang sich nicht in wesentlich stärkerem Grade geltend gemaht hat, als bei den Waren unbekannten Alters. Berücksichtigt man einerseits, daf zum Teil besonders gehaltreiche Waren vorgelegen haben, an denen die Zersetzungsvorgänge, wenn sie nach der Ansicht Jaeckle's in gleicher Richtung sich abspielen würden, besonders deutlich hätten in die Erscheinung treten müssen, andererseits, daf dies tatsächlich nicht der Fall gewesen ist, dann dürfte wohl der von mir gemachte Einwurf der zu schematischen Behandlung durch Jaeckle nicht allein berechtigt, sondern auch wohl begrïndet sein.

Zusammenfassend läßt sich auf Grund der zur Zeit vorliegenden Beobachtungen sagen, daß Jaeckle zwar einen dankenswerten Hinweis auf mögliche Zersetzungsvorgänge, die sich während des Lagerns in der Substanz von Eierteigwaren abspielen und die mit einem Rückgang der Lecithinphosphorsäure verbunden sein können, gegeben hat, ferner, daß dieser sogenannte „Rückgang“ bei gepulvert aufbewahrter Substanz wesentlich schneller verlaufen kann, - nicht aber unbedingt verlaufen muß - als bei unzerkleinertem Material, endlich, daß die Abnahmen an Lecithinphosphorsäure bei Handelswaren innerhalb der Zeit, welche zwischen Herstellung und Konsum in Frage kommt, sich innerhalb derjenigen Grenzen gehalten haben, die der Gutachter bei der Beurteilung bezw. Schätzung des Eigehaltes in Eierteigwaren, so wie so als Spielraum anzunehmen gewohnt ist. Die wahren Ursachen, welche jenen Rückgang bedingen, sind uns noch völlig unbekannt; ebenso läßt sich die Art des Verlanfs der Veränderungen nicht vorhersagen. Alle versuchten Erklärungen erheben sich nicht über das Niveau bloßer Vermutungen, die noch der Aufklärung harren. Weiterem Studium muß es vorbehalten bleiben, Licht in das Dunkel zu bringen. Nach dem augenblicklichen Stande der Dinge muf daran festgehalten werden, daß es möglich ist, den Eigehalt in Eierteigwaren des Handels mit annähernder Sicherheit festzustellen, einerlei ob frische oder ältere Ware vorliegt.

Mai 1905.

\title{
Versuche über Aufnahme von Schwefliger Säure durch Hackfleisch aus den Verbrennungsprodukten des Leuchtgases.
}

Von

\section{A. Kickton.}

Mitteilung aus dem statlichen Hygienischen Institut in Hamburg.

Gelegentlich einer Gerichtsverhandlung, welche infolge einer Beanstandung von Hackfleisch mit einem Gehalt von $0,008 \%$ Schwefliger Säure stattfand, bestritt der 
Angeklagte, dem Hackfleisch ein Schweflige Säure enthaltendes Konservierungsmittel zugesetzt zu haben.

Ein als Sachverständiger anwesender Handelschemiker sagte aus, es sei nicht ausgeschlossen, daß das Hackfleisch aus der Luft Schweflige Säure in geringen Mengen aufnehmen könnte, wenn in dem Aufbewahrungsraum des Fleisches Gasflammen brennen, da das Hamburger Leuchtgas bekanntlich verhältnismäbig stark schwefelhaltig sei. Als Beweis fübrte er einen Prozeß an, in welchem es sich um Lederwaren handelte, die durch Aufnahme ron Sehwefliger Säure bezw. daraus entstandener Schwefelsäure aus den Verbrennungsprodukten des Leuchtgases brüchig geworden seien.

Derselbe Sachverständige führte weiter an, daf nach den „Vereinbarungen zur einheitlichen Untersuchung der Nahrungs- und GenuBmittel" Heft I, S. 36, der Beweis des Zusatzes von scbwefligsauren Salzen erst als erbracht anzusehen sei, wenn $0,1 \%$ Schweflige Säure gefunden werde, während hier noch nicht $1 / 10$ dieses Gehalts festgestellt worden wäre. Auf die Unrichtigkeit des betreffenden Satzes, welcher nur durch einen Irrtum in die Vereinbarungen gelangt sein kann, ist bereits von Beythien in seiner Arbeit über die Verwendung der Schwefligen Säure als Konservierungsmittel ${ }^{1}$ ) hingewiesen worden.

Daßa tatsächlich durch so geringe Mengen von Schwefliger Säure bezw. deren Salzen, wie sie in der in Frage stehenden Probe gefunden wurden, der beabsichtigte Zweck der Erhaltung der roten Farbe des Fleisches bereits erreicht werden kann, haben wir Gelegenheit gehabt festzustellen.

Es lag auch die Möglichkeit vor, daß ein Präservesalz mit einem geringen Gehalt an Schwefliger Säure benutzt worden war, denn uns haben in verschiedenen Fällen als Präservesalze Gemenge von mehreren Salzen vorgelegen, bei welchen der Gehalt an Schwefligsaurem Natrium bis zu $0,3 \%$ herab und selbst noch weniger betrug.

Um die Frage aufzuklären, ob durch das Brennen von Gasflammen in Räumen, in welchem Hackfleisch aufbewahrt wird, Schweflige Säure in nachweisbaren Mengen in das Hackfleisch gelangen kann, wurden auf Anregung von Herrn Dr. Farnst ei $\mathbf{n}$ er folgende Versuche angestellt:

In einem Zimmer von $103 \mathrm{cbm}$ Rauminhalt, also ungefähr entsprechend der Größe eines kleinen Schlächterladens, wurden 4 Bunsenbrenner während 7 Stunden mit nicht leuchtender Flamme brennend erhalten und während dieser Zeit an verschiedenen Stellen des Zimmers Hackfleisch aufgelockert und ausgebreitet offen liegen gelassen. Nach Ablauf der 7 Stunden wurde das Fleisch auf das Vorhandensein von Schwefliger Säure geprüft. In keinem Falle gelang es, in den Proben mittels Kaliumjodat-Stärkepapiers Schweflige Säure nachzuweisen.

Außerdem wurden bei Beginn des Versuchs Kaliumjodat-Stärkepapierstreifen, welche öfter mit einigen Tropfen destillierten Wassers angefeuchtet wurden, frei in dem Raum aufgehängt. Nach einer Stunde trat eine ganz geringe, nur bei durchfallendem Licht sichtbare Bläuung der Papierstreifen ein. Nach 3 Stunden war die Blaufärbung deutlich sichtbar, dann allmählich etwas zunehmend, jedoch nach 7 Stunden immer noch schwach und fast nur an den Rändern des Papiers vorhanden, wie sie bei Spuren von Schwefliger Säure in Hackfleisch zu beobachten ist. Es konnte also bei der Empfindlichkeit der Reaktion verhältnismäßig nur wenig Schweflige Säure sich in der Tuft befinden. Außer der durch die Gasflammen entstandenen Schwef-

1) Diese Zeitschrift $1904,8,43$. 
ligen Säure dürften auch noch andere Bestandteile der umgebenden Luft für die schwache Blaufärbung der Papierstreifen in Frage kommen.

Ferner wurden in einer geschlossenen Kapelle von 1,15 cbm Rauminhalt, bei welcher die Abzugsvorrichtung abgesperrt und nur an einer Seite die Wand am Boden etwa $2 \mathrm{~cm}$ weit für die Luftzufuhr offen gelassen wurde, mehrere aufgelockerte und ausgebreitete, selbst hergestellte Hackfleischproben auf dem Boden und in halber Höhe $(60 \mathrm{~cm})$ offen hingelegt und ein Bunsenbrenner, dessen Gasverbrauch mittels einer eingeschalteten Gasuhr kontrolliert wurde, eine Stunde lang mit nicht leuchtender Flamme gebrannt. Es wurden 951 Leuchtgas verbraucht. Nach Ablauf dieser Zeit gab keine der Fleischproben eine Reaktion auf Schweflige Säure.

Nach weiterem einstündigem Liegenlassen derselben Proben in der geschlossenen Kapelle, während die Gasflamme brannte, war ebenfalls in keiner der Fleischproben Schweflige Säure nachweisbar.

Dieser Versuch wurde in der Weise wiederholt, daß mehrere neue Hackfleischproben in der Kapelle bei brennender Flamme 2 Stunden ohne Unterbrechung offen liegen gelassen und danach auf das Vorhandensein von Schwefliger Säure geprüft wurden. Auch hier ergab sich keine Spur Schwefliger Säure in dem Hackfleisch.

Im Zimmer brannte während dieser Zeit weiter keine Flamme.

Die angegebenen Versuchsbedingungen bei Benutzung der Kapelle würden, auf das ganze Zimmer von $103 \mathrm{cbm}$ Rauminhalt berechnet, einer rund 10- bezw. 20stündigen Brenndauer von 9 Flammen mit einem Gasverbrauch von 951 pro Flamme und Stunde, also im ganzen von rund 8,5 bezw. $17 \mathrm{cbm}$ Leuchtgas entsprechen. Das sind Verhältnisse, wie sie gleich günstig für eine etwaige Aufnahme von Schwefliger Säure durch Hackfleisch aus den Verbrennungsprodukten des Leuchtgases in der Praxis kaum jemals vorkommen dürften. Dabei ist noch zu berücksichtigen, dab in einem Schlächterladen durch häufiges Öffnen der Tür, falls diese, wie im Sommer gewöhnlich, nichl überhaupt offen steht, reichlich für Ableitung der die Verbrennungsprodukte enthaltenden bezw. für Zuführung frischer Luft gesorgt ist.

Durch die Ergebnisse der mitgeteilten Versuche dürfte der Einwand, daß Hackfleisch während der üblichen Aufbewahrungszeit in Räumen, welche durch Gasflammen beleuchtet werden, Schweflige Säure in nachweisbaren Mengen aufnimmt, hinreichend widerlegt erseheinen.

Nach den Versuchen von Dennstedt und A hrens ${ }^{1}$ ) enthält das Hamburger Leuchtgas im Mittel etwa 1,3 g Gesamtschwefel in 10001 Gas. Bei unseren Versuchen würden also in der Kapelle von $1,15 \mathrm{cbm}$ Rauminhalt in einer Stunde bei 951 Gasverbrauch etwa $0,123 \mathrm{~g}$ Schwefel verbrannt bezw. 0,246 g Schweflige Säure und in dem ganzen Zimmer von $103 \mathrm{cbm}$ Rauminhalt bei entsprechendem Gasverbrauch rund $22 \mathrm{~g}$ Schweflige Säure entstanden sein, wenn sämtlicher Schwefel des verbrauchten Leuchtgases durch die Verbrennung in Schweflige Säure übergeführt und diese als solche in der Luft geblieben wäre. Dab aber von dieser verhältnismäßig erheblichen Menge Schwefliger Säure bezw. der doppelten Menge bei zweistündiger Brenndauer in dem Hackfleisch noch nichts nachzuweisen war, dürfte vornehmlich auf eine in kurzer Zeit verlaufende Überführung der durch die Verbrennung des Leuchtgases entstandenen Schwefligen Säure in Schwefelsäure durch den Sauerstoff und Wasserdampf der Luft zurückzufübren sein.

3) Zeitschr. analyt. Chem. 1896, 35, 1. 